\title{
ELLIPTIC CURVES OVER THE RATIONALS WITH BAD REDUCTION AT ONLY ONE PRIME
}

\author{
BAS EDIXHOVEN, ARNOLD DE GROOT, AND JAAP TOP
}

\begin{abstract}
A list is given of elliptic curves over $\mathbf{Q}$ having additive reduction at exactly one prime. It is also proved that for primes congruent to 5 modulo 12 , no such curves having potentially good reduction exist. This enables one to find in a number of cases a complete list of all elliptic curves with bad reduction at only one prime.
\end{abstract}

\section{INTRODUCTION}

This paper grew out of an attempt to find some examples of elliptic curves over $\mathbf{Q}$ with conductor $p^{2}$, for various small primes $p$. The conductor $N$ of an elliptic curve over $\mathbf{Q}$ is an invariant which contains information about the reductions of the curve modulo primes. If the curve has good reduction modulo $p$, then $p$ does not divide $N$; if the curve has multiplicative reduction at $p$, then $p$ divides $N$ exactly once. In case $p \geq 5$ and the curve has additive reduction at $p$, then $p$ divides $N$ exactly twice. Our main result is that in certain cases the only curves with conductor $p^{2}$ are twists of elliptic curves of conductor $p$. In combination with work of Setzer [9] and Brumer and Kramer [2], this allows one to write down for a number of primes $p$ a complete list of the elliptic curves over $\mathbf{Q}$ with good reduction outside $p$. In other cases, an extensive computer search provided us with many examples for primes $p<5000$. These are given in the second half of this paper. There is no guarantee that this list is complete.

\section{Diophantine Results}

Suppose $p$ is a prime number. Our problem is to find all elliptic curves over $\mathbf{Q}$ having additive reduction at $p$ and good reduction at all other primes. Since all elliptic curves with good reduction away from 2 and 3 are known (see e.g. [1, pp. 123-134]), one may assume $p \geq 5$. Given an elliptic curve as desired, one can twist it over the quadratic extension of $\mathbf{Q}$ with discriminant $p$ (compare [10, Chapter $\mathrm{X}, \S \S 2$ and 5$]$ ). The resulting curve has conductor a power of $p$ as well. Moreover, one has:

Lemma 1. Let $p \geq 5$ be a prime number and $K$ the quadratic extension of $\mathbf{Q}$ unramified outside $p$. Up to $K$-twists, all elliptic curves over $\mathbf{Q}$ with good

Received September 12, 1988.

1980 Mathematics Subject Classification (198j Revision). Primary 11G05; Secondary 11D25.

The third author was supported by N.W.O. 
reduction away from $p$ are

1. the ones with multiplicative reduction at $p$;

2. those given by a Weierstrass model with discriminant $\pm p^{2}, \pm p^{3}$, or $\pm p^{4}$. Proof. Given $p$ and $K$ as in the lemma and any elliptic curve $E$ over $\mathbf{Q}$, let $\Delta_{1}$ and $\Delta_{2}$ be the discriminants of Weierstrass models of $E$ and the $K$-twist of $E$, respectively. Then the difference of the valuations at $p$ of $\Delta_{1}$ and $\Delta_{2}$ is congruent to 6 modulo 12. Since any elliptic curve $E$ over $\mathbf{Q}$ has a global Weierstrass minimal model $([10$, p. 226]) and no such curve has everywhere good reduction (e.g. [10, Exercise 8.15]), the lemma follows using the table on p. 46 of [1].

Our main interest is in curves which do not come from the ones with multiplicative reduction at $p$. Curves of conductor $p$ are easily constructed by a method of Mestre [6]. In view of the lemma, this means that we can restrict ourselves to the following case.

Assume one has a Weierstrass model with discriminant $\Delta$, for some $\Delta \epsilon$ $\left\{ \pm p^{2}, \pm p^{3}, \pm p^{4}\right\}$. The $c_{4}$ and $c_{6}$ invariants of this model satisfy the relation $c_{4}^{3}-c_{6}^{2}=1728 \Delta$ ([1, p. 36]). Furthermore, since we are only interested in curves with additive reduction, we assume that $c_{6} \equiv 0 \bmod p$. Then the relation implies that also $c_{4}$ is divisible by $p$. Hence these invariants yield an integral solution of the equation

$$
p X^{3}-Y^{2}=1728 p^{-2} \Delta .
$$

Analyzing this, one obtains:

Proposition 1. If $p$ is a prime number congruent to 5 modulo 12 , then the only integral solutions to

$$
p X^{3}-Y^{2} \in\left\{ \pm 1728, \pm 1728 p, \pm 1728 p^{2}\right\}
$$

are the ones with $Y=0$.

Proof. If $\mathrm{pm}^{3}-n^{2}= \pm 1728$ for some integers $m$ and $n$, then it follows that one of +1728 and -1728 must be a square modulo $p$. If $p \equiv 5 \bmod 12$, neither is, hence the equation has no integral solution in this case.

A similar argument works for $p X^{3}-Y^{2}= \pm 1728 p^{2}$ : given a solution $X=m$ and $Y=n$, one checks that both $m$ and $n$ are divisible by $p$. The integers $m / p$ and $n / p$ satisfy $p^{2}(m / p)^{3}-(n / p)^{2}= \pm 1728$. Again one of \pm 1728 must be a square modulo $p$, which is not the case.

If integers $m, n$ satisfy $p m^{3}-n^{2}= \pm 1728 p$, then it follows that

$$
\mp 3 p\left(\frac{n}{8 \times 3^{2} p}\right)^{2}=\left(\frac{m}{\mp 3 \times 4}\right)^{3}+1 .
$$

Hence we obtain a solution $(x, y) \in \mathbf{Q} \times \mathbf{Q}$ of $d y^{2}=x^{3}+1$, for $d=\mp 3 p$. By an elementary descent argument, Nagell [7, pp. 48-53]) showed already in 1925 that the only rational solution to this is the one with $y=0$. This proves the proposition. 
Remark. In fact, more is true: the descent argument referred to is actually performed for $d y^{2}=x^{3}+1$, where $d$ is any integer not divisible by primes congruent to \pm 1 or 7 modulo 12. (See also Nagell's beautiful review paper on the arithmetic of curves [8, p. 15].) Using the method of descent via twoisogeny, one can easily check this result of Nagell, since one does not come across nontrivial elements in a Shafarevich group (see e.g. [10, pp. 302-304]).

The proposition immediately implies, using the discussion at the beginning of this section:

Theorem 1. If $p$ is a prime number congruent to 5 modulo 12, then every elliptic curve over $\mathbf{Q}$ with conductor $p^{2}$ is a twist of one with conductor $p$.

Corollary 1. For the following primes $p<1000$ no elliptic curve over $\mathbf{Q}$ with good reduction at all places except $p$ exists:

$$
\begin{gathered}
5,29,41,137,173,257,281,293,317,401,449,461,509,521,569, \\
617,641,761,809,821,857,881,929,953,977 .
\end{gathered}
$$

Proof. This follows from pp. 732-733 of [2] and our theorem.

Corollary 2. Suppose $p$ is a prime number of the form $u^{2}+64$ such that $3 \nmid u$. If both the class numbers of $\mathbf{Q}(\sqrt{-p})$ and $\mathbf{Q}(\sqrt{p})$ are not divisible by 3 , then there exists exactly one isogeny class of elliptic curves over $\mathbf{Q}$ with conductor $p^{2}$. This is, e.g., the case for the following five primes:

$$
113,353,3089,4289,9473 .
$$

Proof. Combine our theorem with work of Setzer [9, p. 367].

\section{NUMERICAL RESUltS}

As explained in the previous section, we are interested in finding Weierstrass models over $\mathbf{Q}$ whose $c_{4}$ and $c_{6}$ invariants are related by $c_{4}^{3}-c_{6}^{2}= \pm 1728 p^{N}$, for $2 \leq N \leq 4$. Given integers $c_{4}, c_{6}$ related like this, there is only one elliptic curve over $\mathbf{Q}$ with these invariants, namely the one given by $y^{2}=$ $x^{3}-27 c_{4} x-54 c_{6}$. This model has discriminant $\pm 2^{12} 3^{12} p^{N}$, hence it defines an elliptic curve with conductor $p^{2}$ if and only if it is not minimal at both 2 and 3 . Minimality at 3 is easily checked:

Lemma 2. Suppose $c_{4}$ and $c_{6}$ are integers satisfying $c_{4}^{3}-c_{6}^{2}=1728 d$ with $3 \nmid d$. Take $a$ such that $a \equiv 2 c_{6} \bmod 3$ and $w=\left(a^{3}-2 c_{6}\right) / 3-a c_{4}$. The curve given by $y^{2}=x^{3}-27 c_{4} x-54 c_{6}$ is not minimal at 3 if and only if $a^{2}-c_{4}$ is divisible by 3 and $w$ is divisible by 9 .

This follows, e.g., from Tate's algorithm (see [1, pp. 47-52]). Minimality at 2 is also checked using Tate's algorithm, but requires a little bit more work.

The strategy is now as follows. Given $p$, search for integers $c_{6}$ for which $c_{6}^{2} \pm 1728 p^{N}$ is a cube $(2 \leq N \leq 4)$. (By solving this modulo powers of $p$, one knows in advance the possibilities modulo $p^{2}$ of a solution $c_{6}$, and even modulo $p^{4}$ in case $N=4$.) Having such a $c_{6}$, compute the corresponding $c_{4}$ and check whether the equation $y^{2}=x^{3}-27 c_{4} x \pm 54 c_{6}$ is minimal at 2 and 3 . 
If not, compute a global Weierstrass minimal model corresponding to this. This was done for all (relevant) primes $p$ such that $7 \leq p<5000$ and all values $\left|c_{6}\right| \leq 250000000$. The program found 65 elliptic curves with conductor $p^{2}$, given in the following list:

\begin{tabular}{|c|c|c|c|c|c|c|c|}
\hline$\Delta$ & $a_{1}$ & $a_{2}$ & $a_{3}$ & $a_{4}$ & $a_{6}$ & $c_{4}$ & \\
\hline $7^{3}$ & 1 & -1 & 0 & -37 & -78 & 1785 & 75411 \\
\hline$-7^{3}$ & 1 & -1 & 0 & -2 & -1 & 105 & 1323 \\
\hline$-11^{2}$ & 1 & 1 & 1 & -30 & -76 & 1441 & 54703 \\
\hline$-11^{3}$ & 0 & -1 & 1 & -7 & 10 & 352 & -6776 \\
\hline$-11^{4}$ & 1 & 1 & 0 & -2 & -7 & 121 & 5203 \\
\hline$-19^{3}$ & 0 & 0 & 1 & -38 & 90 & 1824 & -77976 \\
\hline$-37^{2}$ & 1 & -1 & 1 & 2 & -2 & -111 & 999 \\
\hline$-37^{2}$ & 1 & -1 & 1 & -1663 & -25680 & 79809 & 22546431 \\
\hline $37^{3}$ & 0 & 1 & 1 & -12 & -17 & 592 & 10952 \\
\hline $37^{3}$ & 0 & 1 & 1 & -2602 & 50229 & 124912 & -44147512 \\
\hline$-43^{3}$ & 0 & 0 & 1 & -860 & 9707 & 41280 & -8387064 \\
\hline $43^{4}$ & 1 & 0 & 1 & -39 & -27 & 1849 & 20339 \\
\hline$-47^{2}$ & 1 & 1 & 1 & 1 & -2 & -47 & 1927 \\
\hline$-67^{3}$ & 0 & 0 & 1 & -7370 & 243528 & 353760 & -210408408 \\
\hline $79^{3}$ & 1 & -1 & 0 & -64 & -179 & 3081 & 168507 \\
\hline$-157^{3}$ & 1 & 1 & 0 & -42 & 125 & 2041 & -123245 \\
\hline$-163^{3}$ & 0 & 0 & 1 & -2174420 & 1234136692 & 104372160 & -1066294102104 \\
\hline$-179^{2}$ & 1 & -1 & 0 & -11 & -14 & 537 & 14499 \\
\hline$-191^{2}$ & 1 & 0 & 1 & -92 & -345 & 4393 & 291275 \\
\hline $277^{2}$ & 1 & 0 & 0 & -75 & -256 & 3601 & 215783 \\
\hline $307^{2}$ & 1 & -1 & 0 & -19 & -24 & 921 & 24867 \\
\hline$-359^{2}$ & 1 & 0 & 1 & 7 & -15 & -359 & 13283 \\
\hline $397^{2}$ & 1 & -1 & 1 & -174 & 924 & 8337 & -761049 \\
\hline$-397^{3}$ & 1 & 0 & 0 & 91 & 190 & -4367 & -157609 \\
\hline$-431^{4}$ & 1 & 0 & 0 & -3870 & 92773 & 185761 & -80434513 \\
\hline $433^{4}$ & 1 & 0 & 0 & -3906 & -93853 & 187489 & 80807759 \\
\hline$-443^{2}$ & 1 & 0 & 1 & -102 & -403 & 4873 & 340667 \\
\hline$-503^{2}$ & 1 & 0 & 1 & 10 & 21 & -503 & -17605 \\
\hline$-673^{2}$ & 1 & 0 & 0 & -14 & 37 & 673 & -32977 \\
\hline $739^{2}$ & 1 & -1 & 0 & -46 & 127 & 2217 & -99765 \\
\hline$-863^{2}$ & 1 & -1 & 0 & -701 & 7322 & 33657 & -6174765 \\
\hline $877^{3}$ & 0 & -1 & 1 & -2046 & -34926 & 98224 & 30765160 \\
\hline$-887^{2}$ & 1 & 1 & 0 & 19 & -22 & -887 & 2572 \\
\hline$-1069^{2}$ & 1 & 1 & 1 & -824 & -9602 & 39553 & 7999327 \\
\hline$-1103^{2}$ & 1 & 0 & 0 & 23 & -30 & -1103 & 27575 \\
\hline$-1213^{2}$ & 1 & 0 & 1 & -329 & 2265 & 15769 & -1980829 \\
\hline$-1259^{2}$ & 1 & -1 & 1 & -79 & -256 & 3777 & 237951 \\
\hline$-1297^{2}$ & 1 & 1 & 1 & -27 & -94 & 1297 & 71335 \\
\hline$-1367^{2}$ & 1 & 1 & 0 & 29 & 42 & -1367 & -25973 \\
\hline$-1439^{2}$ & 1 & 1 & 1 & 30 & -16 & -1439 & 24463 \\
\hline$-1559^{2}$ & 1 & 0 & 1 & 32 & -21 & -1559 & 20267 \\
\hline$-1607^{2}$ & 1 & 0 & 1 & 33 & 23 & -1607 & -17677 \\
\hline $1753^{2}$ & 1 & 1 & 0 & -36 & -5 & 1753 & -8765 \\
\hline$-1753^{2}$ & 1 & 0 & 1 & -37 & -123 & 1753 & 103427 \\
\hline $1777^{2}$ & 1 & 1 & 1 & -37 & -30 & 1777 & 12439 \\
\hline$-1879^{3}$ & 1 & -1 & 1 & -9043 & 333258 & 434049 & -285981921 \\
\hline
\end{tabular}




$\begin{array}{rrrrrrrr}\Delta & a_{1} & a_{2} & a_{3} & a_{4} & a_{6} & c_{4} & c_{6} \\ -1907^{2} & 1 & 1 & 1 & -257882 & 50298256 & 12378337 & -43550530865 \\ -1993^{2} & 1 & 0 & 1 & -42 & 137 & 1993 & -121573 \\ 1999^{2} & 1 & 1 & 0 & -291 & 1792 & 13993 & -1653173 \\ 2017^{2} & 1 & 1 & 1 & -42 & 22 & 2017 & -34289 \\ 2089^{2} & 1 & 1 & 0 & -43 & -64 & 2089 & 39691 \\ 2251^{2} & 1 & -1 & 1 & -141 & 668 & 6753 & -546993 \\ 2689^{2} & 1 & 1 & 1 & -56 & -120 & 2689 & 83359 \\ 2953^{2} & 1 & 0 & 1 & -62 & -125 & 2953 & 103355 \\ -3203^{2} & 1 & -1 & 0 & -200 & -1051 & 9609 & 951291 \\ -3229^{2} & 1 & 1 & 0 & -10561 & 413370 & 506953 & -360953765 \\ -3313^{2} & 1 & 0 & 0 & -69 & -278 & 3313 & 235223 \\ 3331^{2} & 1 & -1 & 0 & -208 & -1093 & 9993 & 989307 \\ -3469^{2} & 1 & 0 & 1 & -940 & 11007 & 45097 & -9577909 \\ 4129^{2} & 1 & 0 & 0 & -86 & 227 & 4129 & -202321 \\ -4139^{2} & 1 & 1 & 0 & -29921 & 1979690 & 1436233 & -1721223845 \\ -4201^{2} & 1 & 1 & 0 & -87 & 338 & 4201 & -323477 \\ 4423^{2} & 1 & 0 & 0 & -645 & 6248 & 30961 & -5444713 \\ -4513^{2} & 1 & 1 & 1 & -94 & -452 & 4513 & 356527 \\ 4597^{3} & 1 & 1 & 1 & -5842 & -173646 & 280417 & 147926863\end{array}$

It should again be emphasized that there is no reason why this list should be complete. One way to find more examples would be to search for isogenies from the curves in the list to new ones. It turns out that there are no isogenies defined over $\mathbf{Q}$ from curves in our list to new ones, except in the cases where the curve has complex multiplication. In this case, the isogeny is from the curve in our list to its quadratic twist over $\mathbf{Q}(\sqrt{-p})$, so we do not list this isogenous curve. The search for isogenies can be done as follows. Any rational isogeny is built up from rational isogenies of prime degree. Such an isogeny of degree $p$ corresponds to a rational point on the modular curve $X_{0}(p)$. If the genus of this modular curve is zero (which means $p \in\{2,3,5,7,13\}$ ), a good model of it can be found on pp. 238-240 of [6]. In fact we only have to look at integral points on these models, since the curves in our list and those isogenous to them have potentially good reduction, hence integral $j$-invariants (compare [10, Chapter VII, Proposition 5.5 and the proof of Corollary 7.2]). In this way we find three isogenies:

1. The two curves with conductor $7^{2}$ in the list are 2-isogenous; this corresponds to the point $\left(-1,-2^{12}\right)$ on the model $x y=2^{12}$ of $X_{0}(2)$.

2. There is a 7 -isogeny between the curves with discriminant $-37^{2}$; it corresponds to $(-1,-49)$ on the model $x y=49$ of $X_{0}(7)$.

3. Our curves with discriminant $37^{3}$ are 5 -isogenous. This corresponds to the point $\left(1,5^{3}\right)$ on the model of $X_{0}(5)$ given by $x y=5^{3}$.

In case the genus of $X_{0}(p)$ exceeds zero, all rational points are known by work of Mazur [5, pp. 129-130]. It turns out that such points only exist for $p \in\{11,17,19,37,43,67,163\}$. We list the results; they do not give any new examples. 
1. For $p=11$, the isogenies can be found on $\mathrm{p}$. 97 of [1]. The new curves this provides are twists of curves in our table.

2. The curves which admit a rational 17-isogeny are given on p. 80 of [1]. They have multiplicative reduction at 2 .

3. The case $p=37$ is also given on p. 80 of [1]. No twist of these curves has bad reduction at only one prime.

4. The remaining cases correspond to elliptic curves having complex multiplication. As remarked above, they are isogenous to a twist. (Compare $[10$, p. 265].)

Using the methods developed in his thesis [3], B. Edixhoven computed for prime numbers $p<3500$ the number of so-called 'Weil curves' of conductor $p^{2}$ which have potentially supersingular reduction at $p$. He found exactly the same number of such curves as can be found in our list. However, we do not know whether these curves in our list are actually Weil curves.

\section{Complex multiplication CASES}

An excellent reference for the theory of elliptic curves with complex multiplication is [4]. The following result may be classical (compare loc. cit., p. 35, Theorem 12.2.1):

Fact 1. For any imaginary quadratic field $K$ with class number one, there exists an elliptic curve over $\mathbf{Q}$ whose endomorphism ring equals the ring of integers of $K$, and moreover it has good reduction outside the discriminant of $K$.

Of course this can be checked by merely writing down a model as desired for each of the eight well-known $j$-invariants involved. A much more general argument can be found in [4].

The table above contains all the complex multiplication curves (corresponding to imaginary quadratic fields with discriminant $p \geq 5$ ). For convenience they are listed here again (compare p. 84 of [4]).

$\begin{array}{rrrrrrrr}\Delta & a_{1} & a_{2} & a_{3} & a_{4} & a_{6} & c_{4} & c_{6} \\ 7^{3} & 1 & -1 & 0 & -37 & -78 & 1785 & 75411 \\ -7^{3} & 1 & -1 & 0 & -2 & -1 & 105 & 1323 \\ -11^{3} & 0 & -1 & 1 & -7 & 10 & 352 & -6776 \\ -19^{3} & 0 & 0 & 1 & -38 & 90 & 1824 & -77976 \\ -43^{3} & 0 & 0 & 1 & -860 & 9707 & 41280 & -8387064 \\ -67^{3} & 0 & 0 & 1 & -7370 & 243528 & 353760 & -210408408 \\ -163^{3} & 0 & 0 & 1 & -2174420 & 1234136692 & 104372160 & -1066294102104\end{array}$

\section{ACKNOWLEDGMENT}

It is a pleasure to thank F. Oort for his interest in this work and for encouraging us to publish the results.

\section{BIBLIOGRAPHY}

1. B. J. Birch and W. Kuyk (eds.), Modular functions of one variable. IV, Lecture Notes in Math., vol. 476, Springer-Verlag, 1975.

2. A. Brumer and K. Kramer, The rank of elliptic curves, Duke Math. J. 44 (1977), 715-743. 
3. S. J. Edixhoven, Stable models of modular curves and applications, Ph.D. Thesis, Math. Inst., Univ. of Utrecht, 1989.

4. B. H. Gross, Arithmetic on elliptic curves with complex multiplication, Lecture Notes in Math., vol. 776, Springer-Verlag, 1980.

5. B. Mazur, Rational isogenies of prime degree, Invent. Math. 44 (1978), 129-162.

6. J.-F. Mestre, La méthode des graphes. Exemples et applications, Proc. Internat. Conf. on Class Numbers and Fundamental Units of Algebraic Number Fields (Nagoya Univ., Nagoya, 1986), Katata, Japan, 1986, pp. 217-242.

7. T. Nagel, Über die rationalen Punkte auf einigen kubischen Kurven, Tôhoku Math. J. 24 (1925), 48-53.

8. M. T. Nagell, L'analyse indéterminée de degré supérieur, Mémorial des Sciences Mathématiques 39 (1929).

9. B. Setzer, Elliptic curves of prime conductor, J. London Math. Soc. 10 (1975), 367-378.

10. J. H. Silverman, The arithmetic of elliptic curves, Graduate Texts in Math., vol. 106, SpringerVerlag, 1986.

Mathematical Institute, University of Utrecht, Budapestlaan 6, 3508 Ta Utrecht, THE NeTHERLANDS. E-mail: bas@math.berkeley.edu topj@qucdn 